\title{
Uso do Processo Sol-Gel na Obtenção de Materiais Híbridos Organo-Inorgânicos: Preparação, Caracterização e Aplicação em Eletrólitos de Estado Sólido.
}

Rita A. Zoppi e Suzana P. Nunes

Resumo: Neste trabalho é descrita a preparação de materiais híbridos constituídos de um copolímero orgânico contendo segmentos óxido de etileno e de sílica. A rede inorgânica foi formada na solução polimérica a partir da hidrólise e condensação do tetraetoxisilano, TEOS. Como copolímero foi utilizado o poli(óxido de etileno-b-amida-6), PEBAX. Foram preparados também híbridos contendo perclorato de lítio, denominados aqui híbridos eletrólitos. Os materiais foram caracterizados por microscopia eletrônica de transmissão, ensaios de tensão-deformação, análise dinâmico-mecânica e difratometria de raios-X. Os sistemas PEBAX/ $\mathrm{TEOS} / \mathrm{LiClO}_{4}$ foram também caracterizados por espectroscopia de impedância eletroquímica e voltametria cíclica para avaliação de suas propriedades como eletrólito de estado sólido.

Palavras-chave: Processo sol-gel, híbridos, compósitos, eletrólitos poliméricos.

\section{Introdução}

O progresso tecnológico e o desenvolvimento das civilizações dependem, sem dúvida, da obtenção de novos materiais. Individualmente, um número muito reduzido de materiais puros englobam todas as propriedades físicas e mecânicas necessárias para uma determinada aplicação. Assim, materiais puros são modificados de forma que o produto final combine sinergicamente as propriedades de interesse dos constituintes puros. Este sinergismo não depende apenas das propriedades dos componentes individuais, mas também da morfologia das fases que constituem o produto final e das propriedades interfaciais. Uma maneira de se melhorar as interações interfaciais, e consequentemente as propriedades de interesse, é sintetizar materiais que mostrem um alto grau de mistura ou interpenetração entre as fases dissimilares.
Dentro deste contexto, o interesse em usar o processo sol-gel na preparação de materiais híbridos organoinorgânicos tem crescido intensamente devido à possibilidade de se obter uma boa dispersão da fase inorgânica, mesmo a nível molecular, devido ainda às propriedades físicas e químicas que tais híbridos exibem e às possibilidades de processamento oferecidas pelo estado coloidal. A viscosidade dos sóis permite a preparação de filmes por "dip-coating", "spraying" ou "spin-coating". As várias características do processo sol-gel (precursores metalo-orgânicos, solventes orgânicos e baixas temperaturas de processamento) permitem a introdução de moléculas orgânicas "frágeis" dentro da rede inorgânica. Tais moléculas orgânicas podem melhorar as características da matriz, como por exemplo, modificar as propriedades mecânicas, facilitar o processamento de fibras e filmes, possibilitar a moldagem precisa de 
peças para óptica integrada, possibilitar o controle de porosidade e o ajuste do balanço hidrofóbico/ hidrofílico. Além disso, os compostos orgânicos podem conferir uma propriedade peculiar ao material (óptica ou elétrica, eletroquímica, química ou bioquímica). A parte inorgânica do material atua no sentido de melhorar a estabilidade térmica e mecânica, permite a modulação do índice óptico e confere também propriedades magnéticas, elétricas e eletroquímicas interessantes. O processo sol-gel permite a síntese sob condições brandas de novos compostos híbridos organo-inorgânicos com propriedades únicas. Artigos de revisão descrevendo as propriedades desta nova classe de materiais foram recentemente publicados ${ }^{[1,2]}$.

O mecanismo de formação da fase inorgânica via processo sol-gel é bem conhecido e descrito na literatura ${ }^{[3]}$ e se dá a partir da hidrólise e condensação de alcóxidos metálicos principalmente tetrametoxi- ou tetraetoxisilano (TEOS). O procedimento mais simples para a preparação dos híbridos envolve a formação da rede inorgânica em uma solução contendo o polímero orgânico dissolvido.

A investigação de materiais híbridos é uma das áreas de pesquisa que está sendo desenvolvida em nosso laboratório. Híbridos à base de poli(metacrilato de metila) ${ }^{[4]}$, NAFION ${ }^{5]}$, poli(óxido de etileno-coepicloridrina $)^{[6]}$ e borracha de silicona ${ }^{[7]}$ foram preparados e caracterizados. Híbridos constituídos de poli(óxido de etileno-b-amida-6), PEBAX, e sílica obtida a partir da hidrólise e condensação do TEOS também têm sido investigados. Este copolímero é um elastômero e sua combinação com sílica originou materiais com propriedades mecânicas peculiares ${ }^{[8]}$. Ele é também hidrofílico, dissolvendo em solventes polares e favorecendo a dispersão do polímero inorgânico. Ainda devido à sua alta hidroficilidade, o PEBAX tem sido usado na preparação de membranas ${ }^{[9]}$ e a incorporação de sílica pode originar um material com novas propriedades de permeabilidade e seletividade, como já verificado para a borracha de silicona ${ }^{[7]}$.

Uma outra possível aplicação dos materiais híbridos investigados é a preparação de eletrólitos de estado sólido com propriedades mecânicas e de transporte iônico melhoradas. As propriedades do copolímero PEBAX puro (sem sílica) ${ }^{[10]}$ e de sistemas poli(óxido de etileno-co-epicloridrina)/TEOS ${ }^{[6]}$, todos contendo perclorato de lítio, foram recentemente descritas. $\mathrm{Na}$ área de eletrólitos de estado sólido, estudos de complexos de poli(óxido de etileno)/LiX (X sendo um ânion genérico) demonstram claramente que a condução iônica se dá na fase amorfa e que esta depende da mobilidade das cadeias poliméricas. A concentração de portadores de carga depende não só da concentração de sal, mas também do seu grau de dissociação, o qual está relacionado à constante dielétrica do polímero. Diferentes procedimentos de modificação do polímero solvatante são descritos para otimizar as aplicações de eletrólitos sólidos do tipo LiX-polímero à temperatura ambiente ${ }^{[11]}$. A diminuição do grau de cristalinidade e da $\mathrm{T}_{\mathrm{g}}$ pode ser conseguida usando-se métodos químicos (por exemplo, copolimerização do óxido de etileno e seqüências diferentes do óxido de etileno), ou usando-se métodos de modificação estrutural (por exemplo, reticulação química ou física do poli(óxido de etileno)) e físicos (por exemplo, a utilização de plastificantes) ${ }^{[11]}$. Com respeito aos materiais híbridos, a rede de sílica, além de poder melhorar as propriedades térmicas e mecânicas, contribui para aumentar a fração amorfa no sistema. A formação de um híbrido pode ser um procedimento alternativo para modificar as propriedades do polímero e para melhorar a condutividade iônica de eletrólitos poliméricos. Sistemas do tipo NAFION/TEOS/TMDES (TMDES $=1,1,3,3$ tetrametil-1,3-dietoxidisiloxano) foram recentemente caracterizados por espectroscopia de impedância eletroquímica com o propósito de se avaliar o efeito da presença de segmentos siloxanos mais flexíveis na parte inorgânica do material sobre a condutividade iônica do híbrido ${ }^{[12]}$.

Neste trabalho serão apresentados resultados que mostram a influência das diferentes condições de preparação na morfologia e nas propriedades mecânicas dos sistemas PEBAX/TEOS. Serão apresentados também resultados referentes à preparação de uma nova classe de eletrólitos de estado sólido: os híbridos eletrólitos PEBAX/TEOS/ $/ \mathrm{LiClO}_{4}$.

\section{Experimental}

\section{Preparação dos Híbridos PEBAX/TEOS}

O poli(óxido de etileno-b-amida-6) (PEBAX MX1657) foi fornecido pela Elf Acquitaine. Sua composição química foi confirmada por análise elementar feita em um analisador elementar CHN Perkin Elmer 2400, resultando em $40 \%(\mathrm{~m} / \mathrm{m})$ de blocos de poliamida-6 e $60 \%(\mathrm{~m} / \mathrm{m})$ de blocos de poli(óxido de etileno). 
Uma vez que um dos blocos é uma poliamida, o copolímero é solúvel em poucos solventes. Neste trabalho foram utilizadas soluções do polímero em ácido fórmico:tetraidrofurano 1:1 e em n-butanol.

Diferentes volumes de tetraetoxisilano (Aldrich) foram adicionados a uma solução de ácido fórmico:tetraidrofurano 1:1/PEBAX $10 \%(\mathrm{~m} / \mathrm{m})$ mantida sob agitação, com $\mathrm{pH}=1$. Diferentes quantidades de água foram adicionadas à solução, sempre mantendo uma razão molar água/TEOS de 2:1. Manteve-se a solução (cerca de $30 \mathrm{ml}$ ) sob agitação por $8 \mathrm{~h}$ a temperatura ambiente e transferiu-se a mesma para placas de Petri de $10 \mathrm{~cm}$ de diâmetro, as quais foram mantidas fechadas por $16 \mathrm{~h}$ para permitir que a reação continuasse. As placas foram abertas e o solvente foi evaporado a $50^{\circ} \mathrm{C}$. Os filmes obtidos foram secos sob vácuo durante uma semana.

O mesmo procedimento foi usado para uma solução de n-butanol/PEBAX 3\% (m/m), com pH = 7 . Soluções mais concentradas sofreram gelificação e não puderam ser obtidas com este solvente. Neste caso, ao invés de água, diferentes quantidades de uma solução aquosa de $\mathrm{HCl} 0,15 \mathrm{M}$ foram adicionadas para manter a razão molar água/TEOS igual a 2:1. A solução foi mantida sob agitação por $6,5 \mathrm{~h}$ a $60^{\circ} \mathrm{C}$ e transferida para placas de TEFLON, as quais foram mantidas fechadas por $16 \mathrm{~h}$. O solvente foi evaporado a $50^{\circ} \mathrm{C}$ e os filmes foram secos sob vácuo durante uma semana.

\section{Preparação dos Hibridos Eletrólitos PEBAX/TEOS/LiCIO}

Neste caso, seguiu-se o mesmo procedimento descrito para a preparação dos híbridos de PEBAX/ TEOS obtidos a partir da solução do polímero em nbutanol. Diferentes quantidades de $\mathrm{LiClO}_{4}$ foram adicionadas à solução (n-butanol/PEBAX contendo TEOS e $\mathrm{HCl} 0,15 \mathrm{M}$ ) antes de sua transferência para as placas de TEFLON. Após a completa dissolução do sal, a solução foi transferida para placas de TEFLON, as quais permaneceram fechadas por $16 \mathrm{~h}$. $\mathrm{O}$ solvente foi evaporado a $50^{\circ} \mathrm{C}$ e os filmes foram secos sob vácuo durante uma semana.

\section{Ensaios de Tensão-Deformação}

Ensaios de tensão-deformação foram feitos em uma máquina universal de ensaios Emic Mem 200 a uma velocidade de separação das garras igual a $500 \mathrm{~mm}$. $\mathrm{min}^{-1}$ e usando-se uma cela de carga de $500 \mathrm{~N}$. Foram analisados 15 espécimens de cada amostra com dimensões iguais a 10x0,70x50mm. Os dados mostrados nas Tabelas 1 e 2 correspondem à $(M \pm s)$, onde $M$ é a média aritmética e $s$ é o desvio padrão.

\section{Análise Dinâmico-Mecânica}

A análise dinâmico-mecânica foi feita em um analisador térmico TA Instruments DMA 983, na faixa de -150 a $200^{\circ} \mathrm{C}$, a uma frequência de $1 \mathrm{~Hz}$ e amplitude de $0,20 \mathrm{~mm}$.

\section{Difratometria de raios- $X$}

Medidas de difração de raios- $X$ foram feitas em um difratômetro de raios-X Shimadzu XD-3A, usando a radiação de $\mathrm{CuK} \alpha$.

\section{Microscopia Eletrônica de Transmissão}

As amostras foram cortadas em um crioultramicrótomo Leitz-Reichert-Jung FC4E, a $-80^{\circ} \mathrm{C}$, com uma faca de diamante. Os filmes finos (espessura aproximada de $70 \mathrm{~nm}$ ) foram observados em microscópio eletrônico de transmissão Zeiss CEM-902.

\section{Espectroscopia de Impedância Eletroquímica}

Medidas de impedância eletroquímica foram feitas em uma câmara seca sob argônio. Os filmes foram prensados entre dois eletrodos de aço inoxidável (diâmetro igual a $1 \mathrm{~cm}$; área igual a $0,785 \mathrm{~cm}^{2}$ ) e fixados em uma cela rosqueável construída em náilon. As medidas foram feitas usando-se um analisador de resposta de frequência Schlumberger Solartron $1255 \mathrm{HF}$ acoplado a um potenciostato PAR 273 interfaceado a um computador. A faixa de frequência analisada foi $10^{-1}-10^{5} \mathrm{~Hz}$, e a de temperatura foi de $25 \mathrm{a} 100^{\circ} \mathrm{C}$.

\section{Voltametria Cíclica}

A voltametria cíclica foi feita em uma câmara seca sob argônio. Os filmes foram prensados entre dois eletrodos, um de aço inoxidável e o outro de lítio metálico na forma de placa (diâmetro igual a $1 \mathrm{~cm}$; área igual a $0,785 \mathrm{~cm}^{2}$ ), e fixados em uma cela rosqueável construída em náilon. As medidas foram feitas usando-se um potenciostato PAR 273 interfaceado a um computador, e registradas em um

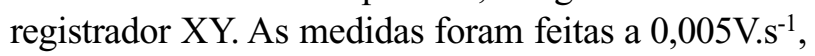
a $80^{\circ} \mathrm{C}$, numa faixa de 0 a $5,5 \mathrm{~V}\left(v s . \mathrm{Li}^{\circ}\right)$. 


\section{Resultados e Discussão}

\section{Sistemas Hibridos PEBAX/TEOS}

A Figura 1 mostra as curvas de tensão-deformação dos filmes de PEBAX/TEOS com composição igual a 100:0, 75:25 e 60:40. Outras composições foram também analisadas. As Tabelas 1 e 2 mostram os valores de módulo de elasticidade, tensão de ruptura, deformação de ruptura e energia de ruptura para os híbridos preparados em ácido fórmico:THF 1:1 ou em n-butanol, em função do teor de TEOS no material. Em ambos os casos (materiais preparados em ácido fórmico:THF e em butanol), observa-se um aumento do módulo de elasticidade com o aumento da quantidade de TEOS, ou seja, os materiais tornam-se mais rígidos com o aumento do teor de componente inorgânico, como esperado.

Com relação a tensão de ruptura e a energia de ruptura, embora muito mais evidente para os híbridos preparados em ácido fórmico:THF 1:1, foram observados em ambos os casos (materiais preparados em ácido fórmico:THF e em butanol) valores máximos das duas grandezas quando o teor de TEOS foi menor ou igual a $33 \%$.

Uma diferença de comportamento bastante significativa foi observada com relação aos valores de deformação de ruptura obtidos. Para os híbridos preparados em n-butanol, a deformação de ruptura diminui gradualmente com o aumento da quantidade de TEOS no material. Ao contrário, os híbridos preparados em ácido fórmico:THF 1:1 apresentaram valores máximos de deformação de ruptura quando o teor de TEOS foi igual a $25 \%$.

O comportamento mecânico dos híbridos pode ser explicado fazendo-se uma analogia com o efeito da adição de cargas rígidas particuladas nas propriedades de tensão-deformação de polímeros. Quando

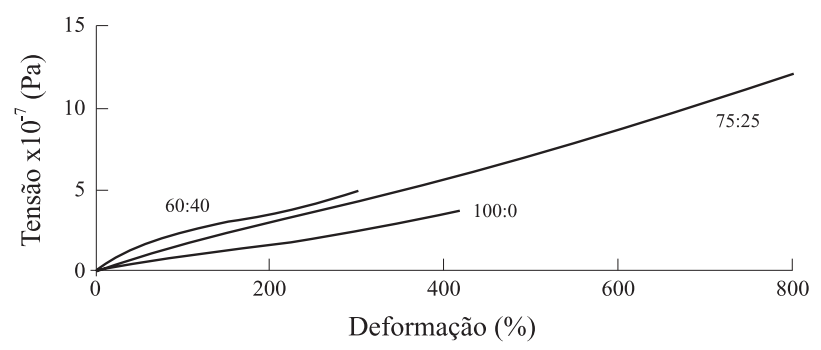

Figura 1. Curvas de tensão-deformação de híbridos de PEBAX/TEOS preparados em ácido fórmico:THF 1:1, com composição igual a 100:0, $75: 25$ e $60: 40$.
Tabela 1. Valores do módulo de elasticidade, tensão de ruptura, deformação de ruptura e energia de ruptura de híbridos de PEBAX/ TEOS preparados em n-butanol.

\begin{tabular}{ccccc}
\hline $\begin{array}{c}\text { PEBAX/ } \\
\text { TEOS }\end{array}$ & $\begin{array}{c}\text { Módulo de } \\
\text { elasticidade } \\
(\mathrm{MPa})\end{array}$ & $\begin{array}{c}\text { Tensão de } \\
\text { ruptura } \\
(\mathrm{MPa})\end{array}$ & $\begin{array}{c}\text { Deformação } \\
\text { de ruptura } \\
(\%)\end{array}$ & $\begin{array}{c}\text { Energia de } \\
\text { ruptura }(\mathrm{J})\end{array}$ \\
\hline $100: 0^{\mathrm{a}}$ & $37 \pm 5$ & $34 \pm 6$ & $330 \pm 35$ & $0,52 \pm 0,09$ \\
$78: 22^{\mathrm{a}}$ & $55 \pm 5$ & $43 \pm 16$ & $309 \pm 39$ & $0,8 \pm 0,2$ \\
$75: 25^{\mathrm{a}}$ & $56 \pm 8$ & $40 \pm 11$ & $304 \pm 34$ & $0,7 \pm 0,1$ \\
$71: 29^{\mathrm{a}}$ & $50 \pm 5$ & $4 \pm 7$ & $300 \pm 31$ & $1,0 \pm 0,1$ \\
$67: 33^{\mathrm{a}}$ & $50 \pm 7$ & $39 \pm 10$ & $287 \pm 33$ & $1,0 \pm 0,2$ \\
$60: 40^{\mathrm{a}}$ & $62 \pm 6$ & $23 \pm 7$ & $168 \pm 28$ & $0,4 \pm 0,1$ \\
$100: 0^{\mathrm{b}}$ & $49 \pm 9$ & $0,8 \pm 0,2$ & $1,8 \pm 0,5$ & $(2,6 \pm 0,8) \times 10^{-4}$ \\
\hline
\end{tabular}

${ }^{\mathrm{a}} \mathrm{pH}=7 \quad$ b $\mathrm{pH}=1$

Tabela 2. Valores do módulo de elasticidade, tensão de ruptura, deformação de ruptura e energia de ruptura de híbridos de PEBAX/ TEOS preparados em ácido fórmico:THF 1:1, a temperatura ambiente.

\begin{tabular}{ccccc}
$\begin{array}{c}\text { PEBAX/ } \\
\text { TEOS }\end{array}$ & $\begin{array}{c}\text { Módulo de } \\
\text { elasticidade } \\
(\mathrm{MPa})\end{array}$ & $\begin{array}{c}\text { Tensão de } \\
\text { ruptura } \\
(\mathrm{MPa})\end{array}$ & $\begin{array}{c}\text { Deformação } \\
\text { de ruptura } \\
(\%)\end{array}$ & $\begin{array}{c}\text { Energia de } \\
\text { ruptura (J) }\end{array}$ \\
\hline $100: 0$ & $12 \pm 1$ & $40 \pm 12$ & $469 \pm 97$ & $1,9 \pm 0,3$ \\
$78: 22$ & $15 \pm 2$ & $77 \pm 6$ & $679 \pm 92$ & $1,9 \pm 0,3$ \\
$75: 25$ & $16 \pm 1$ & $151 \pm 42$ & $904 \pm 178$ & $3,0 \pm 0,6$ \\
$71: 29$ & $18 \pm 2$ & $53 \pm 7$ & $434 \pm 80$ & $1,6 \pm 0,2$ \\
$67: 33$ & $21 \pm 6$ & $41 \pm 8$ & $352 \pm 62$ & $1,8 \pm 0,5$ \\
$60: 40$ & $28 \pm 5$ & $33 \pm 13$ & $267 \pm 78$ & $1,3 \pm 0,5$ \\
$100: 0^{*}$ & $28 \pm 4$ & $20 \pm 1$ & $137 \pm 12$ & $0,9 \pm 0,1$ \\
\hline
\end{tabular}

*Filmes obtidos a partir de soluções em ácido fórmico:THF 1:1 aquecidos previamente por $6,5 \mathrm{~h}$ a $60^{\circ} \mathrm{C}$.

existe uma boa adesão entre a carga rígida e o polímero, sua presença resulta num aumento do módulo de elasticidade. Geralmente, tais cargas causam uma diminuição da tensão de ruptura, mas existem numerosas exceções (por exemplo, negro de fumo em borrachas). Elas causam normalmente uma diminuição da deformação de ruptura, como observado para os híbridos preparados em n-butanol. Entretanto, se a presença de tais cargas introduzirem rachadura ("crazing") adicional, e ao mesmo tempo, se elas atuarem como inibidores de propagação da fratura, é possível que o polímero contendo cargas rígidas apresente valores de deformação de ruptura iguais ou maiores que os observados para o polímero não carregado $^{[13]}$, como no caso dos híbridos preparados em ácido fórmico:THF 1:1.

Comparando os dados de tensão-deformação obtidos para os híbridos preparados em ácido fórmico:THF 1:1 $(\mathrm{pH}=1)$ e em n-butanol $(\mathrm{pH}=7)$, independentemente da composição, verificou-se que 
os materiais preparados em n-butanol apresentaram módulos de elasticidade maiores, deformação de ruptura e energia de ruptura menores, e valores de tensão de ruptura próximos (com excessão de 22 e 25\%) dos observados para os híbridos preparados em ácido fórmico:THF. Mesmo os filmes de PEBAX puro obtidos a partir da solução do polímero nos dois solventes apresentaram propriedades distintas. A Tabela 3 mostra os valores de grau de cristalinidade dos híbridos preparados a partir dos dois solventes. Aqueles preparados em n-butanol são significativamente mais cristalinos que os materiais preparados em ácido fórmico:THF 1:1. No caso particular dos híbridos, além do grau de cristalinidade, aspectos adicionais devem ser considerados, pois durante a preparação em ácido fórmico e em n-butanol, foram diferentes também o $\mathrm{pH}$ e a temperatura.

Conforme descrito em um artigo anterior ${ }^{[8]}$, para analisar a importância da acidez, híbridos de PEBAX/ TEOS foram preparados em n-butanol ajustando-se o $\mathrm{pH}$ da solução de n-butanol/PEBAX para 1 (usou-se $\mathrm{HCl}$ concentrado para o ajuste) ou 7. Tanto os filmes puros como os híbridos obtidos a partir da solução com $\mathrm{pH}=$ 1 foram muito quebradiços (Tabela 1). Filmes de PEBAX puro obtidos a partir de soluções de n-butanol/ PEBAX com diferentes valores de $\mathrm{pH}$ foram analisados por espectroscopia de infravermelho. A razão de intensidade entre as bandas em $1106 \mathrm{~cm}^{-1}$ (característica do estiramento $\mathrm{C}-\mathrm{O}-\mathrm{C}$ no bloco de poli(óxido de etileno)) e $1544 \mathrm{~cm}^{-1}$ (característica da deformação N-H e estiramento $\mathrm{C}-\mathrm{N}$ no bloco de poliamida-6) mudou de $1,00 \mathrm{em} \mathrm{pH}=7$, para $1,20 \mathrm{em} \mathrm{pH}=1$, confirmando que em soluções ácidas os grupos amida hidrolisam formando grupos amina e ácido carboxílico ${ }^{[8,9]}$, podendo originar filmes muito frágeis mecanicamente. Para analisar

Tabela 3- Grau de cristalinidade de híbridos de PEBAX/TEOS preparados em ácido fórmico:THF 1:1 ou em n-butanol em função da composição.

\begin{tabular}{ccc}
\hline & \multicolumn{2}{c}{ Grau de Cristalinidade (\%) } \\
\cline { 2 - 3 } PEBAX/TEOS & $\begin{array}{c}\text { ácido fórmico: } \\
\text { THF } 1: 1\end{array}$ & n-butanol \\
\hline $100: 0$ & 7 & 56 \\
$78: 22$ & 4 & 47 \\
$75: 25$ & 3 & 49 \\
$71: 29$ & 3 & 55 \\
$67: 33$ & 4 & 51 \\
$60: 40$ & 3 & 42 \\
\hline
\end{tabular}

o efeito da temperatura, foram preparados filmes de PEBAX puro a partir da solução ácido fórmico:THF 1:1/PEBAX a temperatura ambiente ou a $60^{\circ} \mathrm{C}$ (Tabela 2). Os filmes preparados a $60^{\circ} \mathrm{C}$ foram mais quebradiços, no entanto, o efeito da temperatura foi muito menos acentuado comparado ao efeito do $\mathrm{pH}$.

Na Figura 2 são mostradas as curvas de LogE' em função da temperatura para filmes de PEBAX puro obtidos a partir das soluções de n-butanol/PEBAX ou ácido fórmico:THF 1:1/PEBAX. O efeito do grau de cristalinidade é bastante evidente nestes casos. Para ambos os filmes, observa-se o início de uma região de transição em aproximadamente $-20^{\circ} \mathrm{C}$ com uma diminuição gradual do módulo de armazenamento. Próximo de $30^{\circ} \mathrm{C}$, o módulo diminui mais drasticamente e finalmente atinge um "plateau". A magnitude desse "plateau" é maior para amostras com grau de cristalinidade mais altos (amostras obtidas em nbutanol). Normalmente os cristalitos presentes atuam como pontos de reticulação física, aumentando o módulo e inibindo o escoamento do polímero. Próximo de $200^{\circ} \mathrm{C}$, em ambos os casos observa-se o fenômeno de escoamento como uma consequência da fusão.

A Figura 3 mostra o comportamento dinâmicomecânico de híbridos de PEBAX/TEOS com diferentes composições, preparados em ácido fórmico:THF 1:1. O comportamento observado para as amostras preparadas em n-butanol foi similar ao observado na Figura 3. As curvas de LogE' em função da temperatura apresentaram a mesma tendência descrita para os filmes de PEBAX puro (Figura 3a). A magnitude do "plateau" entre o final da região de transição e o início da fusão foi maior para amostras contendo maiores teores de TEOS. De um modo ge-

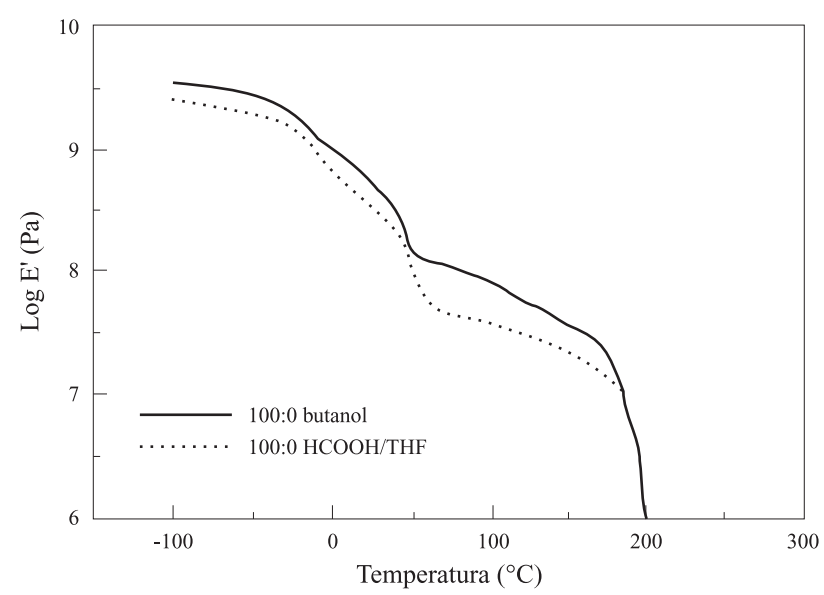

Figura 2- Logaritmo do módulo de armazenamento em função da temperatura para filmes de PEBAX puro preparados em (.......) ácido fórmico:THF 1:1 ou em ( - ) n-butanol. 

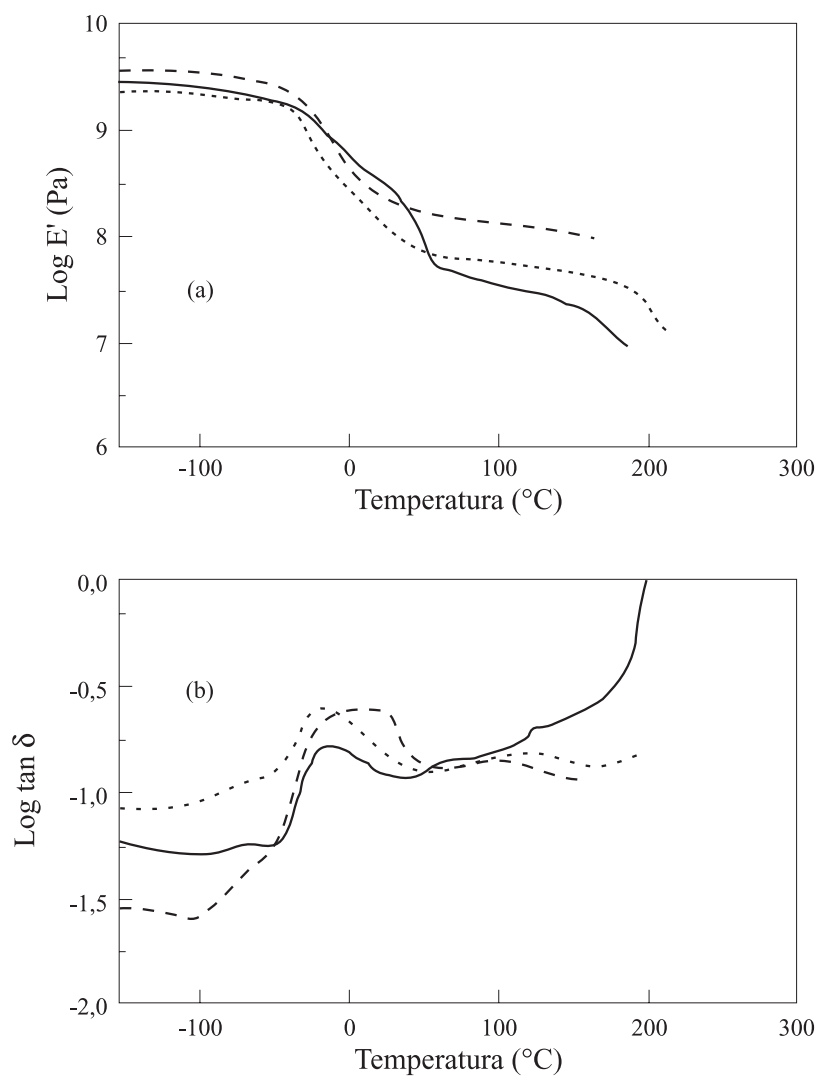

Figura 3. a) Logaritmo do módulo de armazenamento em função da temperatura de híbridos de PEBAX/TEOS preparados em ácido fórmico:THF 1:1 com composição (_- 100:0, (......) 75:25 e (- - - -) 60:40. b) Logaritmo da tangente de delta em função da temperatura de híbridos de PEBAX/TEOS preparados em ácido fórmico:THF 1:1 com composição (—) 100:0, (......) 75:25 e (- - - 60:40.

ral, isto é observado também em amostras de polímero contendo cargas rígidas particuladas ${ }^{[13]}$. Considerando as curvas de LogTanઈ em função da temperatura (Figura 3b), observa-se que a incorporação de sílica causou um alargamento do pico associado à temperatura de transição vítrea do material (aproximadamente $-20^{\circ} \mathrm{C}$ ). Esse alargamento pode ser associado aos diferentes processos de relaxação que ocorrem em polímeros contendo cargas rígidas particuladas e pode também indicar a presença de microheterogeneidades no sistema, decorrente de uma segregação de fases ${ }^{[13]}$.

Para verificar a ocorrência de segregação de fases, as amostras foram analisadas por microscopia eletrônica de transmissão, Figura 4. Os filmes ultrafinos (70nm de espessura) foram observados em um microscópio que possui um espectrômetro de perda de energia de elétrons. Se apenas os elétrons espalhados elasticamente são selecionados, obtém-se uma imagem de campo claro com aberração cromática reduzida. Se os elétrons espalhados inelasticamente são seleciona- dos, são formadas imagens com contraste elementar mostrando a concentração local de um elemento específico. Na Figura 4 são mostradas as micrografias obtidas selecionando-se elétrons espalhados elasticamente para filmes de PEBAX puro e filmes de PEBAX/TEOS com composição igual a 60:40, preparados em ácido fórmico:THF 1:1 ou em n-butanol. Morfologias similares foram observadas para os híbridos de outras composições. Para as amostras preparadas em ácido fórmico:THF, observou-se uma morfologia típica de polímeros cristalinos com estruturas fibrilares. Para as amostras preparadas em n-butanol foram observadas estruturas globulares dispersas numa matriz mais clara. A composição dessas estruturas globulares foi analisada usando-se o espectrômetro de perda de energia de elétrons. Foi detectada uma concentração local de nitrogênio, tanto para os filmes de PEBAX puro como para os híbridos de PEBAX/TEOS. Independentemente da composição e do solvente usado na preparação, não foram observados domínios de sílica nas amostras dos híbridos, mostrando que a sílica pode estar bem distribuída na matriz ou que os domínios de sílica são menores que o limite de detecção da técnica. Além disso, as diferenças observadas na morfologia concernem à estrutura supermolecular e não refletem as diferenças de grau de cristalinidade. Como mostrado na Tabela 3, as amostras preparadas em n-butanol apresentaram maiores graus de cristalinidade que aquelas preparadas em ácido fórmico:THF 1:1. Diferentes tipos de estrutura (globular e fibrilar) foram observadas para filmes de poliamida obtidos a partir de diferentes solventes ${ }^{[14]}$. Filmes de poliamida obtidos a partir de uma solução de glicerina/poliamida mostraram uma morfologia do tipo globular, enquanto que os filmes preparados a partir de uma solução de ácido fórmico/ poliamida apresentaram uma morfologia do tipo fibrilar $^{[14]}$. As soluções de PEBAX em n-butanol, apesar de menos concentradas ( $3 \% \mathrm{~m} / \mathrm{m})$, são muito mais viscosas e normalmente formam um gel à temperatura ambiente. A alta viscosidade pode ter desfavorecido a formação de estruturas supermoleculares ordenadas semelhantes àquelas observadas para os filmes preparados a partir da solução de ácido fórmico:THF/ PEBAX.

\section{Híbridos Eletrólitos PEBAX/TEOS/LiClO ${ }_{4}$}

A Figura 5 mostra a condutividade iônica dos sistemas PEBAX/TEOS/ $/ \mathrm{LiClO}_{4}$ em função das concentrações de sal e de TEOS, medida a $40^{\circ} \mathrm{C}$. O mesmo 

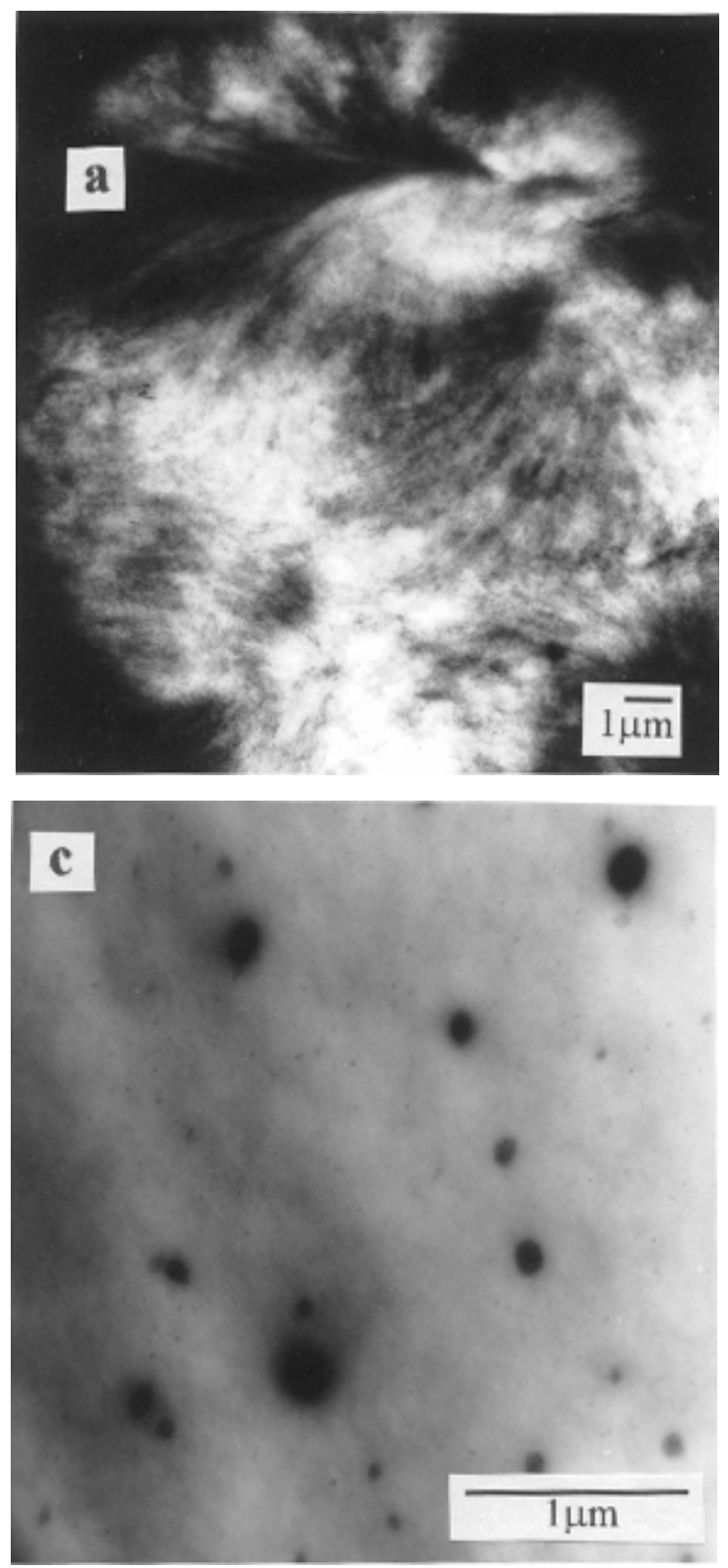
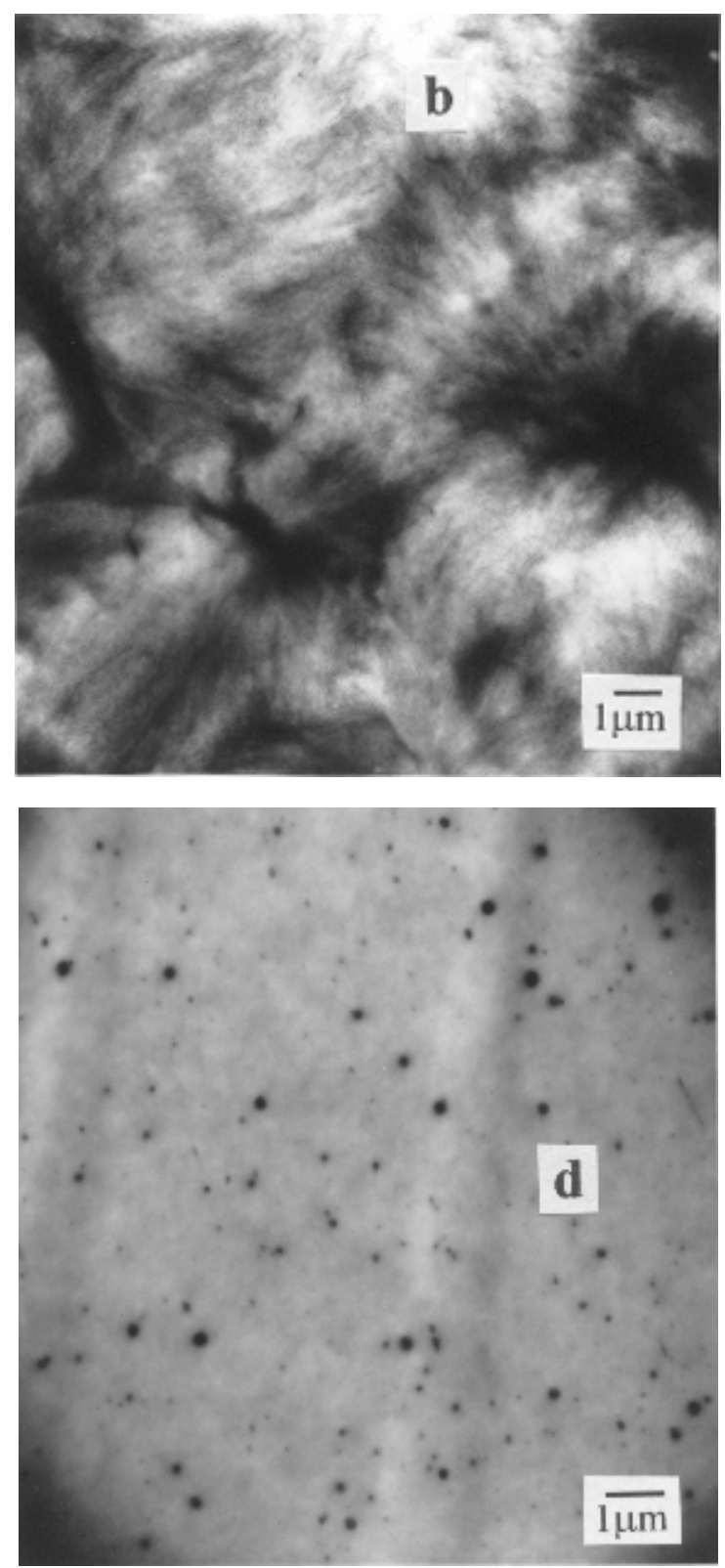

Figura 4- Microscopia eletrônica de transmissão de filmes de (a, c) PEBAX puro e de híbridos de (b, d) PEBAX/TEOS com composição 60:40 preparados em $(\mathrm{a}, \mathrm{b})$ ácido fórmico:THF 1:1 ou em $(\mathrm{c}, \mathrm{d}) \mathrm{n}$-butanol.

comportamento foi observado quando as medidas foram feitas em temperaturas na faixa de 25 a $100^{\circ} \mathrm{C}$. Foram obtidos valores máximos de condutividade iônica para concentrações de sal e de TEOS intermediárias. A dependência da condutividade com a concentração de sal no caso dos híbridos eletrólitos foi similar àquela observada para os sistemas PEBAX/ $\mathrm{LiClO}_{4}$ sem sílica ${ }^{[10]}$, e tem sido associada a dois efeitos opostos. $\mathrm{O}$ aumento da condutividade na região de baixas concentrações de sal pode ser atribuído ao aumento do número de portadores de carga; a diminuição da condutividade na região de altas concen- trações de sal pode ser atribuída a uma diminuição da mobilidade dos portadores de carga causada pelo enrijecimento das cadeias do polímero solvatante, onde a complexação iônica (polímero- $\mathrm{Li}^{+}$) atuaria como pontos de reticulação ${ }^{[15]}$.

A Figura 6 mostra a dependência da condutividade iônica com a temperatura para os híbridos eletrólitos de PEBAX/TEOS/ $/ \mathrm{LiClO}_{4}$ contendo $50 \%$ de TEOS e $15 \%$ de $\mathrm{LiClO}_{4}$. Comportamentos similares foram observados para as outras composições. Nota-se uma boa concordância dos dados experimentais com a equação de Vogel-Tamman-Fulcher, equação de VTF (li- 


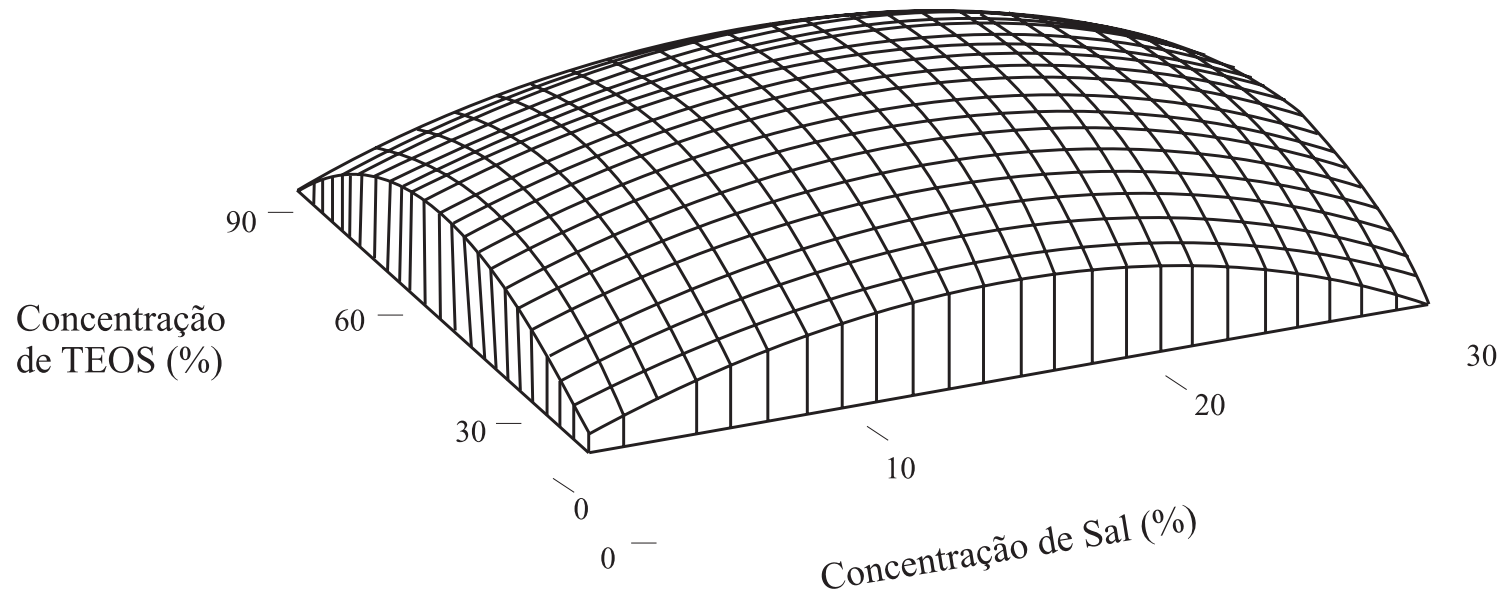

Figura 5. Condutividade iônica de $\mathrm{PEBAX} / \mathrm{TEOS} / \mathrm{LiClO}_{4}$ em função do teor de sal e de TEOS, a $40^{\circ} \mathrm{C}$.

nha cheia $)^{[15]}$. Um dos parâmetros que pode ser obtido do ajuste das curvas experimentais com a equação de VTF é a energia de ativação aparente necessária para a redistribuição de volume livre, diretamente relacionada à energia de ativação para o processo de condução iônica ${ }^{[15]}$, Tabela 4. Os híbridos eletrólitos apresentaram valores de energia de ativação sempre menores comparados aos obtidos para os sistemas PEBAX/ $\mathrm{LiClO}_{4}$ sem sílica. Este mesmo comportamento foi também verificado nos sistemas constituídos de poli(óxido de etileno-co-epicloridrina) recentemente investigados ${ }^{[6]}$. Os menores valores de energia de ativação observados para os híbridos eletrólitos pode estar relacionado à formação de um sistema rico em grupos silanóis gerados pela hidrólise do alcóxido me-

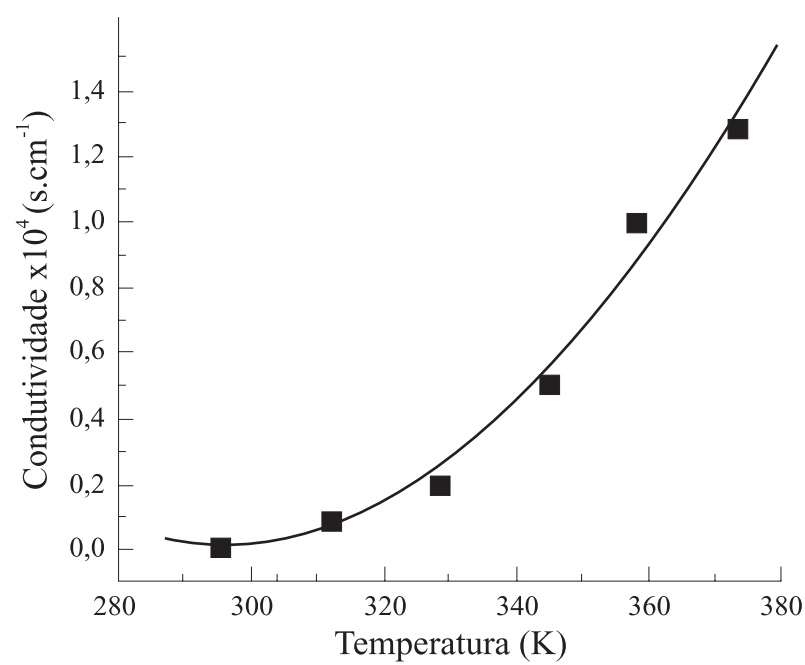

Figura 6. Condutividade iônica de $\mathrm{PEBAX} / \mathrm{TEOS} / \mathrm{LiClO}_{4}$ em função da temperatura (teor de sal $=15 \%$; teor de TEOS $=50 \%$ ). A linha cheia representa o ajuste dos dados experimentais com a equação de VogelTamman-Fulcher ${ }^{[15]}$. tálico, que facilitariam o processo de condução. Assim, seria possível considerar até a ocorrência de um mecanismo de condução diferente incluindo o transporte iônico pelos segmentos silanóis.

A Figura 7 mostra as curvas de LogE' e de LogTan $\delta$ em função da temperatura de híbridos eletrólitos de PEBAX/TEOS/LiClO 4 contendo $15 \%$ de sal e 15 ou $50 \%$ de TEOS. Nota-se que a magnitude do "plateau" que aparece depois da região de transição (entre 25 e $100^{\circ} \mathrm{C}$ aproximadamente) nas curvas de LogE', é menor para os híbridos eletrólitos contendo 50\% de TEOS. A temperatura de transição vítrea do material, associada ao pico próximo de $0^{\circ} \mathrm{C}$ nas curvas de $\log T a n \delta$, é também menor para as amostras contendo $50 \%$ de TEOS. A análise por difratometria de raios-X das amostras confirmou que os sistemas PEBAX/TEOS/ $/ \mathrm{LiClO}_{4}$ contendo maiores teores de TEOS são menos cristalinos, o que conferiria ao material maior flexibilidade, resultando em menores valores de LogE'. Nos sistemas PEBAX/TEOS/ $/ \mathrm{LiClO}_{4}$, dois efeitos opostos estariam influenciando o comportamento do módulo de armazenamento: com o aumento da concentração de TEOS, a rigidez deveria aumentar também, mas o grau

Tabela 4. Valores de energia de ativação aparente de PEBAX/TEOS/ $\mathrm{LiClO}_{4}$ e de PEBAX/LiClO 4 em função da concentração de sal. O teor de TEOS nos híbridos eletrólitos é igual a $50 \%$.

\begin{tabular}{|c|c|c|c|}
\hline \multicolumn{2}{|c|}{ PEBAX/TEOS/LiClO ${ }_{4}$} & \multicolumn{2}{|c|}{ PEBAX/LiClO ${ }_{4}^{*}$} \\
\hline $\begin{array}{c}\text { Teor de Sal } \\
(\%)\end{array}$ & $\begin{array}{c}\Delta \mathrm{E} \\
\left(\mathrm{kJ} \cdot \mathrm{mol}^{-1}\right)\end{array}$ & $\begin{array}{l}\text { Teor de Sal } \\
\quad(\%)\end{array}$ & $\begin{array}{c}\Delta \mathrm{E} \\
\left(\mathrm{kJ} \cdot \mathrm{mol}^{-1}\right)\end{array}$ \\
\hline 1 & 13,4 & 1 & 16 \\
\hline 15 & 9,7 & 15 & 37 \\
\hline 30 & 11,1 & 20 & 50 \\
\hline
\end{tabular}

*Valores obtidos da referência[10] 


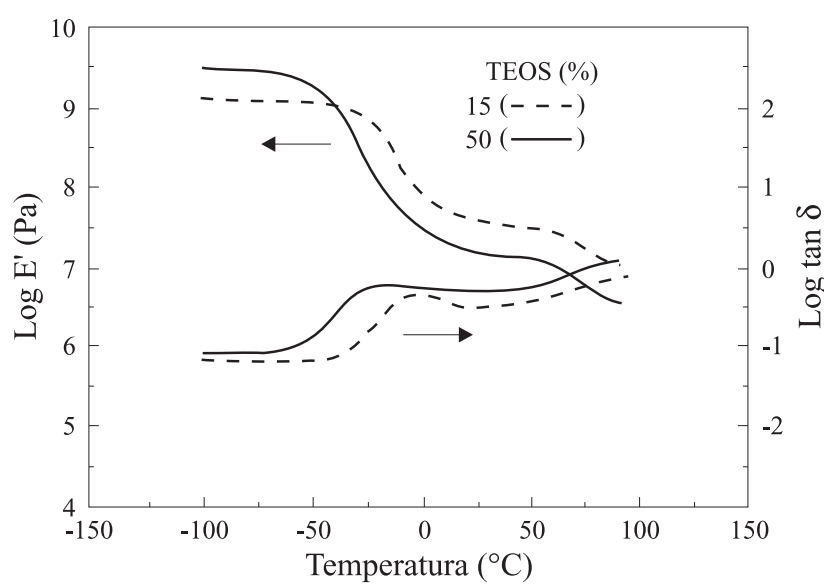

Figura 7. Logaritmo do módulo de armazenamento e da tangente de delta em função da temperatura de híbridos eletrólitos de PEBAX/TEOS/ $\mathrm{LiClO}_{4}$ contendo 15 ou $50 \%$ de TEOS (teor de sal $=15 \%$ ).

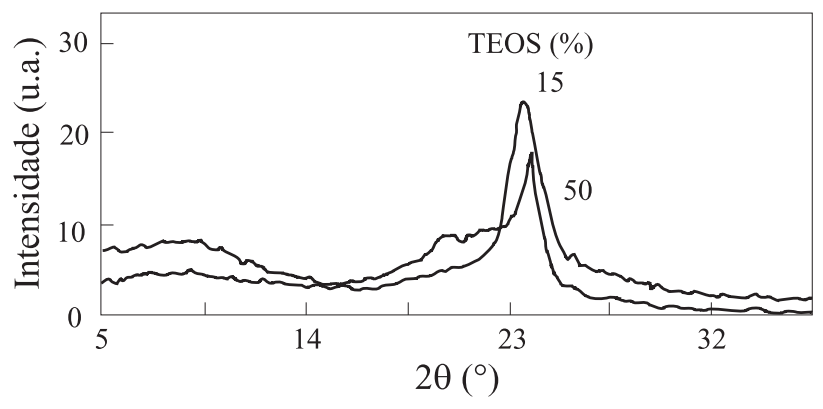

Figura 8- Difratogramas de raios- $X$ de híbridos eletrólitos de PEBAX/ TEOS $/ \mathrm{LiClO}_{4}$ contendo 15 ou $50 \%$ de TEOS (teor de sal $=15 \%$ ).

de cristalinidade diminui, contribuindo no sentido de aumentar a flexibilidade. Nos híbridos eletrólitos contendo $50 \%$ de TEOS, o efeito da cristalinidade foi predominante e a flexibilidade desses filmes foi maior que a dos sistemas contendo $15 \%$ de TEOS. No entanto, é importante ressaltar que amostras contendo teores de TEOS iguais a $85 \%$ foram mais rígidas e quebradiças. A magnitude do "plateau" que aparece depois da região de transição nas curvas de LogE' de filmes de PEBAX/ $\mathrm{LiClO}_{4}$ sem sílica (concentração de sal $=15 \%$ ), é da mesma ordem de grandeza daquela observada para os híbridos eletrólitos contendo 50\% de TEOS e 15\% de sal. Então, pode-se dizer que a incorporação de pequenas quantidades de sílica (por exemplo, 15\% de TEOS) não influencia significativamente o grau de cristalinidade e pode promover o reforço mecânico do material.

A Figura 9 mostra a voltametria cíclica do sistema aço inoxidável/híbrido eletrólito/ $/ i^{\circ}$, feita a $80^{\circ} \mathrm{C}$ sob atmosfera de argônio. O filme de PEBAX/TEOS/ $\mathrm{LiClO}_{4}$ analisado contém $15 \%$ de sal e $50 \%$ de TEOS. A janela de estabilidade eletroquímica foi aproximadamente igual a $4 \mathrm{~V}($ de 0,5 a $4,5 \mathrm{~V})$. Na extremidade catódica, a janela eletroquímica é limitada pelo pro-

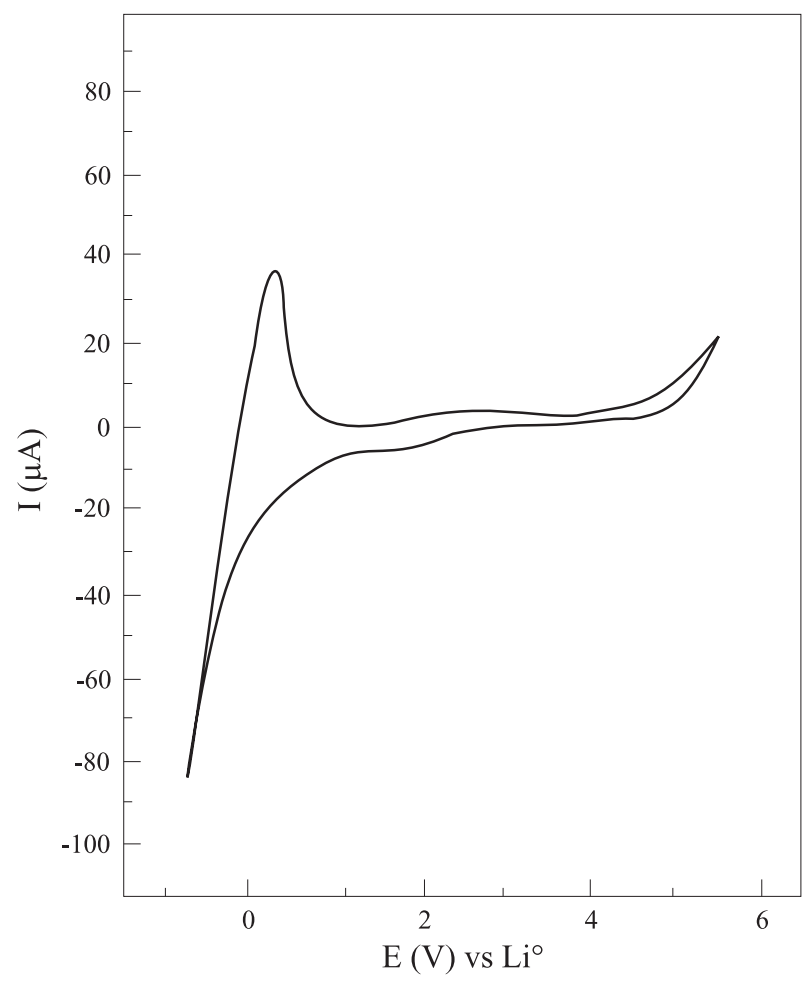

Figura 9. Voltametria cíclica de PEBAX/TEOS/ $/ \mathrm{LiClO}_{4}$ contendo $50 \%$ de TEOS e $15 \%$ sal. Velocidade de varredura $=0,005 \mathrm{~V} \cdot \mathrm{s}^{-1} ;$ temperatura $=80^{\circ} \mathrm{C}$.

cesso de deposição do lítio e na extremidade anódica, a faixa de estabilidade é limitada pelo processo de oxidação do ânion ${ }^{[15]}$. O mesmo comportamento foi observado para os eletrólitos $\mathrm{PEBAX} / \mathrm{LiClO}_{4} \mathrm{sem}$ sílica $^{[10]}$, mostrando que a incorporação do componente inorgânico parece não afetar a janela de estabilidade eletroquímica do sistema.

\section{Conclusões}

Híbridos organo-inorgânicos de PEBAX e sílica foram preparados a partir da hidrólise e condensação do TEOS. Usando-se solventes diferentes para o polímero, foram observadas drásticas mudanças de morfologia e do grau de cristalinidade. Como resultado, foram observados também diferentes comportamentos nos ensaios dinâmico-mecânicos e de tensão-deformação. Independentemente das condições de síntese, a incorporação de sílica promoveu um enrijecimento do polímero orgânico, confirmado pelo aumento do módulo de elasticidade com o aumento da concentração de TEOS. Sob condições específicas (sistema PEBAX/TEOS 75:25 preparado em ácido fórmico:THF), foi possível obter materiais com módulo de elasticidade e tenacidade maiores com- 
parados ao polímero puro.

A incorporação de sais de lítio aos híbridos permitiu a obtenção de uma nova classe de eletrólitos de estado sólido. A condutividade iônica dos sistemas PEBAX/TEOS/LiClO 4 foi da mesma ordem de grandeza que aquela observada para os eletrólitos de $\mathrm{PEBAX} / \mathrm{LiClO}_{4}$ sem sílica $\left(\sigma \cong 10^{-5} \mathrm{~S}_{\mathrm{cm}} \mathrm{cm}^{-1}\right.$ a $\left.25^{\circ} \mathrm{C}\right)$. A principal vantagem da incorporação de sílica nos eletrólitos poliméricos usando-se o processo sol-gel foi diminuir a energia de ativação para o transporte iônico.

\section{Agradecimentos}

Os autores agradecem à FAPESP e ao CNPq. Rita A. Zoppi agradece a bolsa concedida pela FAPESP (Proc. 94/2161-9).

\section{Referências Bibliográficas}

1. D.A. Loy e K.J. Shea; "Bridged polysilsesquioxanes. Hybrid porous hybrid organic-inorganic materials", Chem. Rev., 95, 1431-1442, (1995).

2. U. Schubert, N. Husing e A. Lorenz; "Hybrid inorganic-organic materials by sol-gel processing of organofunctional metal alkoxides", Chem. Mater., 7, 2010-2027, (1995).

3 C.J. Brinker; "Hydrolysis and condensation of silicates: effects on structure", J. NonCrystalline Solids, 100, 31-50, (1988).

4. K.F. Silveira, I.V.P. Yoshida e S.P. Nunes; "Phase separation in PMMA/silica sol-gel systems", Polymer, 36, 1425-1434, (1995).

5. R.A. Zoppi, I.V.P. Yoshida e S.P. Nunes; "Hybrids of perfluorosulfonic acid ionomer and silicon oxide by sol-gel reaction from solution. Morphology and thermal analysis", Polymer, no prelo.
6. R.A. Zoppi, C.M.N.P. Fonseca, M.-A. De Paoli e S.P. Nunes; "Hybrid electrolytes of poly(ethylene oxide) copolymers $/ \mathrm{LiClO}_{4} / \mathrm{SiO}_{2}$ : thermal analysis, mechanical properties and chemometric study of ionic conductivity", Acta Polymer., 48, 131-136, (1997).

7. S.P. Nunes, J. Schultz e K.V. Peinemann; “Silicone membranes with silica nanoparticles", J. Mater. Sci. Lett., 15, 1139-1141, (1996).

8. R.A. Zoppi, C.R. Castro, I.V.P. Yoshida e S.P. Nunes; "Hybrids of $\mathrm{SiO}_{2}$ and poly(amide 6-b-ethylene oxide)", Polymer, 38, 5705-5712, (1997).

9. S.P. Nunes, M.L. Sforça e K.V. Peinemann; "Dense hydrophilic composite membranes for ultrafiltration", J. Membrane Sci., 106, 49-56, (1995).

10. R.A. Zoppi, C.M.N.P. Fonseca, M.-A. De Paoli e S.P. Nunes; "Solid electrolytes based on poly(amide 6-b-ethylene oxide)", Solid State Ionics, 91, 123-130, (1996).

11. M. Gauthier, M. Armand e D. Muller; "Aprotic polymer electrolytes and their applications" in "Electroresponsitive Molecular and Polymeric Systems", T.A. Skotheim ed., Marcel Dekker Inc., Nova Iorque, vol. 1, (1988).

12. R.A. Zoppi e S.P. Nunes; "Hybrids of perfluorosulfonic acid ionomer and silicon oxide by sol-gel reaction from solution. Electrochemical impedance spectroscopy studies", J. Electroanal. Chem., no prelo.

13. L.E. Nielsen; "Mechanical properties of polymers and composites", Marcel Dekker Inc., Nova Iorque, (1974).

14. H.G. Elias; "Macromolecules", Plenum Press, Nova Iorque, vol.1, (1977).

15. C.A. Vincent; "Polymer electrolytes", Prog. Solid St. Chem., 17, 145-261, (1987). 